\title{
THE AGE OF THE GALACTIC INNER HALO
}

\author{
LAURA KELLAR FULLTON AND BRUCE W. CARNEY \\ Dept. of Physics \& Astronomy, University of North Carolina \\ CB \# 3255 Phillips Hall, Chapel Hill, NC 27599-3255
}

Are the inner regions of the Galaxy younger, older or the same age as the outer halo? We have undertaken a study of globular clusters which lie within 4-5 kpc of the Galactic center. Here we present results for three of these clusters: NGC 6723, NGC 6352 and NG.C 5927. We have constructed cluster color-magnitude diagrams from which we have determined their ages using the magnitude difference between the horizontal branch and main-sequence turnoff following the procedure of Carney, Storm \& Jones (1992, CSJ). We find that 47 Tuc, NGC 6352 and NGC 6723 are coeval to within the errors, while NGC 5927 appears younger. The figure below is a reproduction of Figure 20 of CSJ with our clusters added.

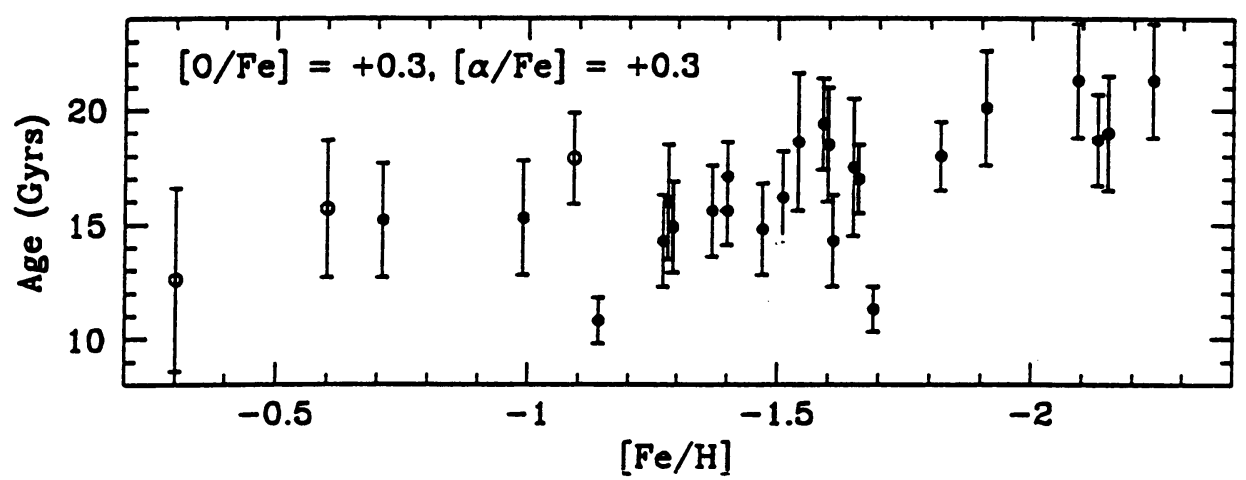

Figure 1. Reproduction of Figure 20 of CSJ with clusters from this poster added (open symbols). 